\title{
Main active constituent identification in Guanxinjing capsule, a traditional Chinese medicine, for the treatment of coronary heart disease complicated with depression
}

\author{
Yan-qiong ZHANG, \#, Qiu-yan GUO 1, \#, Qiu-yue LI, ${ }^{1, \#}$, Wei-qiong REN ${ }^{2}$, Shi-huan TANG ${ }^{1}$, Song-song WANG ${ }^{3}$, Ri-xin LIANG $^{1}$, \\ De-feng $\mathrm{LI}^{1}$, Yi ZHANG ${ }^{1}$, Hai-yu XU ${ }^{1, *}$, Hong-jun YANG ${ }^{1}$ \\ ${ }^{1}$ Institute of Chinese Materia Medica, China Academy of Chinese Medical Sciences, Beijing 100700, China; ${ }^{2}$ The First Affiliated \\ Hospital of Hunan University of Traditional Chinese Medicine, Changsha 410007, China; ${ }^{3}$ State Key Laboratory of Quality Research in \\ Chinese Medicine, Institute of Chinese Medical Sciences, University of Macau, Macau 999078, China
}

\begin{abstract}
Guanxinjing capsules (GXJCs) are used in traditional Chinese medicine as a common therapy for coronary heart disease (CHD) complicated with depression. In this study, we aimed to identify the main active constituents in GXJCs and to investigate the mechanisms of GXJC action on CHD complicated with depression. The chemical constituent profile of the GXJC was identified by UHPLC-LTQ-Orbitrap assay, and oral bioavailability was evaluated to screen the GXJC drug-like chemical constituents. A total of 16 GXJC drug-like chemical constituents were identified. Then, putative targets of the GXJC drug-like chemical constituents were predicted using MedChem Studio, with 870 genes found to be the putative targets of these molecules. After that, a GXJC putative target-known $\mathrm{CHD}$ /depression therapeutic target network was constructed, and four topological features, including degree, betweenness, closeness and K-coreness, were calculated. According to the topological feature values of the GXJC putative targets, 14 main active constituents were identified because their corresponding putative targets had topological importance in the GXJC putative target-known CHD/ depression therapeutic target network, which were defined as the candidate targets of GXJC against CHD complicated with depression. Functionally, these candidate targets were significantly involved in several CHD/depression-related pathways, including repairing pathological vascular changes, reducing platelet aggregation and inflammation, and affecting patient depression. This study identified a list of main active constituents of GXJC acting on CHD complicated with depression using an integrative pharmacology-based approach that combined active chemical constituent identification, drug target prediction and network analysis. This method may offer an efficient way to understand the pharmacological mechanisms of traditional Chinese medicine prescriptions.
\end{abstract}

Keywords: traditional Chinese medicine; Guanxinjing capsule; coronary heart disease; depression; integrative pharmacology; in silico ADME prediction; target prediction; network analysis

Acta Pharmacologica Sinica (2018) 39: 975-987; doi: 10.1038/aps.2017.117; published online 31 Aug 2017

\section{Introduction}

Coronary heart disease (CHD), as one of the leading causes of mortality worldwide, has been indicated as a main cause for approximately thirty percent of deaths in people under sixtyfive years old and nearly twenty-five percent of all deaths ${ }^{[1]}$. Despite the recent advances in therapeutic strategies for this disease, the morbidity and mortality of CHD patients remains

\footnotetext{
\# These authors contributed equally to this work.

${ }^{*}$ To whom correspondence should be addressed.

E-mail hyxu@icmm.ac.cn

Received 2017-03-15 Accepted 2017-05-19
}

high. While its incidence is falling in the majority of developed countries, such as the United States and the United Kingdom, it is still increasing in most developing countries including Singapore and other countries in Asia ${ }^{[2]}$. According to the declaration of the National Heart Foundation of Australia, depression has been indicated as an important independent risk factor for CHD in terms of etiology or prognosis ${ }^{[3,4]}$. Growing evidence shows that the prevalence of depression in CHD patients is very high. It has been reported that the rate of major depressive disorder may be approximately fifteen percent after myocardial infarction (MI) or coronary artery bypass grafts. Once milder forms of depression are included, 
the prevalence may be greater than forty percent ${ }^{[5,6]}$. Although there are many types of therapeutic strategies used to treat patients with CHD complicated with depression ${ }^{[7]}$, such as pharmacological therapy (eg, fluoxetine, sertraline, citalopram and mirtazapine), psychological therapy (eg, CBT and interpersonal psychotherapy) and collaborative care, patient outcomes remain unsatisfying due to the uncertain therapeutic efficacies, unbearable high prices of treatment and unavoidable adverse effects of these treatments.

To address this problem, more and more patients are attempting to seek efficient prevention and therapy from traditional Chinese medicine (TCM), which is a crucial part of complementary and alternative medicine ${ }^{[8]}$. Characterized by its holistic concept, TCM usually attenuates physical and mental dysfunctions as a whole. Growing clinical evidence shows the efficiency of TCM in the treatment of chronic diseases with few side effects, especially for patients with CHD complicated with depression ${ }^{[9-11]}$. Guan Xing Jing Capsule (GXJC), which is an important Chinese patent medicine, is widely used in the treatment of $\mathrm{CHD}$ complicated with depression. According to the theory of TCM, CHD belongs to the thoracic obstruction category and is defined as a kind of psychosomatic disorder. Our previous study demonstrated that ' $q i$ ' deficiency, blood stasis, the lack of spirit and 'Shen' preservation are all the main causes of $\mathrm{CHD}^{[12]}$. GXJC is composed of Radix Salviae miltiorrhizae, Radix Paeoniae rubra, Ligusticum chuanxiong, Carthamus tinctorius L, Rhizome of Fragrant Solomon's Seal, Panax notoginseng, Panax ginseng root, Storax, and Borneol, all of which are prepared according to the Chinese pharmacopeia ${ }^{[13]}$. Growing clinical evidence shows that GXJC may possess multiple therapeutic effects including supplementing ' $q i$ ', activating blood circulation, tranquilizing 'Shen', sedating 'Zhi' and balancing psychosomatic status; thus, it may be of vital importance and promotional value in the prophylaxis and treatment of CHD. Moreover, accumulating clinical observations have indicated that GXJC can also effectively relieve symptoms of CHD complicated with depression and improve quality of patients' lives without any significant side effects ${ }^{[14-16]}$. Pharmacologically, GXJC has been revealed to exert its therapeutic effects on psycho-cardiology mainly through anti-myocardial ischemia, resistance to anoxia and anti-depression ${ }^{[16]}$. However, the underlying mechanisms of GXJC action on CHD complicated with depression remain unclear.

Therefore, in the current study, the chemical constituent profile containing GXJC was identified by UHPLC-LTQ-Orbitrap assay. Oral bioavailability was evaluated to screen for GXJC drug-like chemical constituents. Then, putative targets of the GXJC drug-like chemical constituents were predicted using MedChem Studio. Subsequently, the GXJC putative target-known CHD/depression therapeutic target network was constructed, and four topological features, including degree, betweenness, closeness and k-coreness, were calculated to identify the key active constituents of GXJC that act on CHD complicated with depression as well as to investigate its underlying pharmacological mechanisms. A schematic diagram of this study is illustrated in Figure 1.

\section{Materials and methods}

\section{Data collection of chemical constituents in GXJC}

The names, molecular formulas, molecular weights, and 2D and 3D structures of the chemical constituents of interest including Radix Salviae miltiorrihizae, Radix Paeoniae rubra, Ligusticum chuanxiong, Carthamus tinctorius L, Rhizome of Fragrant Solomon's Seal, Panax notoginseng, Panax ginseng root, Storax, and Borneol were obtained from PubMed ${ }^{[17]}$ (https:// www.ncbi.nlm.nih.gov/pubmed/, 2014-02-21), SinoMed (http://sinomed.cintcm.ac.cn/index.jsp, 2014-03-12), Chemistry Database of the Chinese Academy of Sciences ${ }^{[18]}$ (http:/ / www.organchem.csdb.cn, 2014-03-05) and TCM Database@ Taiwan, China ${ }^{[19]}$ (http://tcm.cmu.edu.tw/, updated on 201402-28). Of the 838 constituents collected from the above databases, 197 were for Radix Salviae miltiorrhizae, 22 for Radix Paeoniae rubra, 96 for Ligusticum chuanxiong, 99 for Carthamus tinctorius L, 28 for rhizome of Fragrant Solomon's Seal, 184 for Panax notoginseng, 192 for Panax ginseng root, 32 for Storax and 16 for Borneol. Detailed information about the chemical constituents containing GXJC is provided in Supplementary Table S1.

\section{Instrument and UHPLC-LTQ-Orbitrap conditions LC system}

A Thermo Accela ultra-high-pressure liquid chromatography (UHPLC) system (Thermo Fisher Scientific, San Jose, CA, USA) was used to analyze all samples with a flow rate of 0.3 $\mathrm{mL} / \mathrm{min}$ and an injection volume of $2 \mu \mathrm{L}$. Chromatographic separation was carried out using a reverse-phase column Syncronis C18 column (Thermo Fisher Scientific, San Jose, CA, USA) at $35^{\circ} \mathrm{C}$. The mobile phases $\mathrm{A}$ and $\mathrm{B}$ consisted of $0.1 \%$ formic acid in water and acetonitrile, respectively. The mobile phase gradient program was as follows: $0.0-15.0 \mathrm{~min}: 5 \%-32 \%$ B; $15.0-20.0$ min: $32 \%-52.5 \%$ B; $20.0-35.0$ min: $52.5 \%-95 \%$ B. The on-line UV spectra ranged from $200 \mathrm{~nm}$ to $400 \mathrm{~nm}$.

\section{Mass spectrometry and data processing}

The LC-ESI-MS ${ }^{\mathrm{n}}$ experiments were performed using a Thermo Fisher LTQ-Orbitrap Velos Pro Hybrid mass spectrometer (Thermo Fisher Scientific, Bremen, Germany) equipped with an electrospray ionization (ESI) source. Among the parameters of the ESI source, sheath and auxiliary gas flow were set to 35 and 10 arbitrary units, respectively; capillary temperature was $350{ }^{\circ} \mathrm{C}$; spray voltage was $5 \mathrm{kV}$ in the positive model and $4.5 \mathrm{kV}$ in the negative model; and S-LensRF level was 62.02. The following mass spectra were acquired in the mass range of $100-1200 \mathrm{~m} / \mathrm{z}$. The scan cycle employed a full-scan event at a resolution of 30000 (at $\mathrm{m} / \mathrm{z} 400$ ) and three data-dependent $\mathrm{MS}^{\mathrm{n}}$ events acquired at a resolving power of 15000 . The collision energy for collision-induced dissociation (CID) was adjusted to $35 \%$ of the maximum. All parameters were identical to those used in the LC-MS ${ }^{\text {n }}$ experiments. Data analyses were performed using Mass Frontier 6.0 (Thermo Fisher Scientific) software and Xcalibur 2.1 (Thermo Fisher Scientific) software. Considering the possible elemental compositions of potential constituents present in the GXJC samples, the numbers and types 


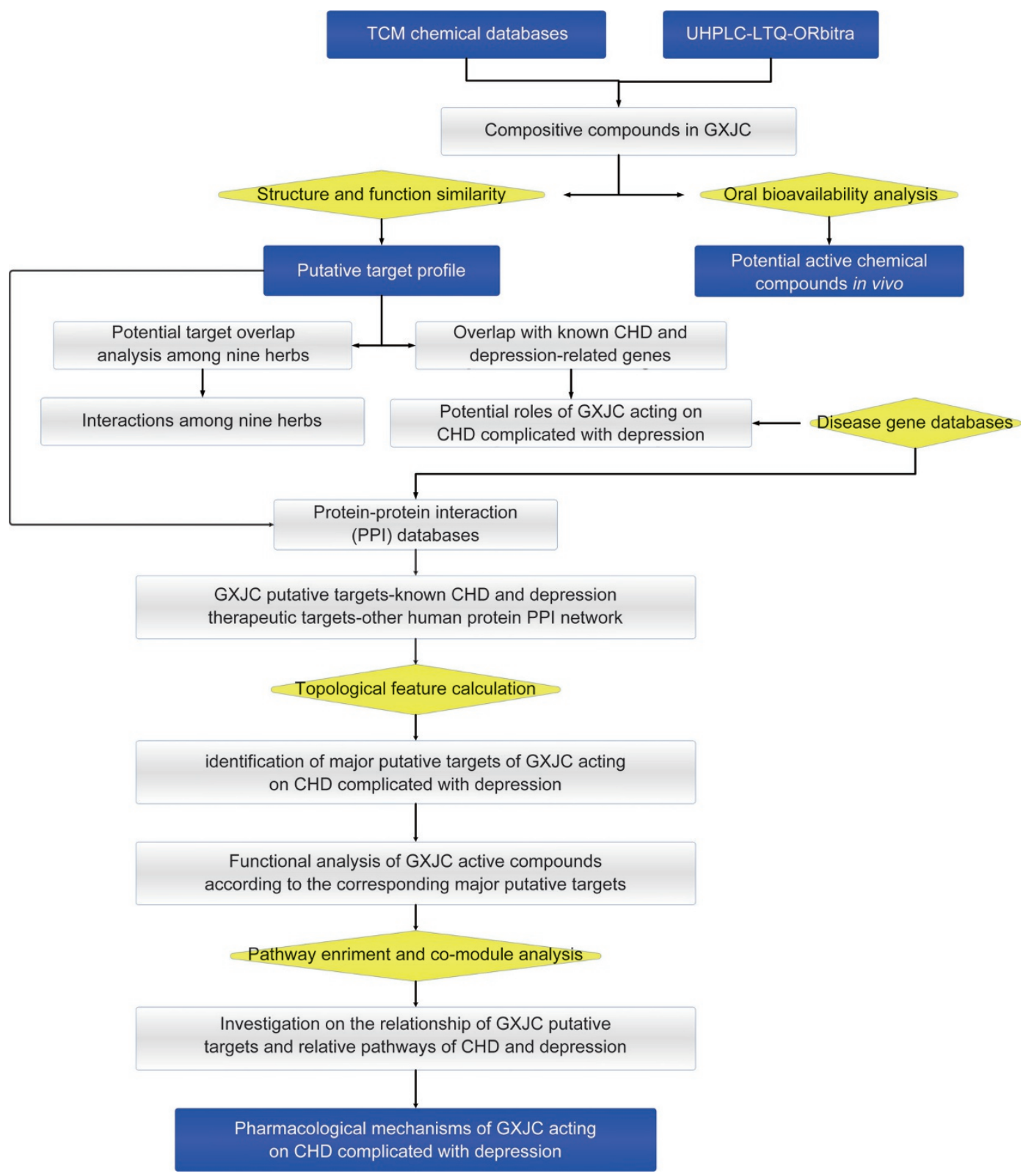

Figure 1. A schematic diagram of the integrative pharmacology-based strategies to identify key active constituents and molecular mechanisms of Guanxinjing capsule acting on psycho-cardiology.

of expected atoms were set as follows: carbon $\leq 100$, hydrogen $\leq 250$, oxygen $\leq 60$, and nitrogen $\leq 10$. The accuracy error threshold was fixed at $10 \mathrm{ppm}$.

Prediction of absorbed constituents and oral bioavailability using in silico absorption, distribution, metabolism, and excretion (ADME) evaluation models

Structural information files $\left({ }^{*}\right.$.mol or *.sdf files) related to the GXJC constituents (identified by UHPLC-LTQ-Orbitrap) were downloaded from ChemSpider ${ }^{[20]}$ (http://www.chemspider. com, 2014-04-12). ADME evaluation of these constituents was employed to predict the apparent permeability coefficient (Papp) and oral bioavailability (OB) using ACD/Percepta software $5.07^{[21]}$ (ACD/Labs, Toronto, Canada), including the pas- sive intestinal permeability of the Caco-2 module and the PK explorer module. The papp and $\mathrm{OB}$ of one constituent were greater than $9.0 \times 10^{6} \mathrm{~cm} / \mathrm{s}$ and $40 \%$, respectively, which indicated good drug-likeness.

\section{Network construction and analysis}

Known therapeutic targets for the treatment of CHD and depression

Known therapeutic targets for the treatment of CHD and depression were collected from Drugbank database ${ }^{[22]}$ (www. drugbank.ca/, v3.0) and the OMIM database ${ }^{[23]}$ (www.omim. org, last updated 2013-10-31). Detailed information on the collected known therapeutic targets for these two diseases is provided in Supplementary file S1-section 1 and Supplementary 
Tables S2, S3.

\section{Drug target prediction for GXJC}

The putative targets of GXJC were predicted using MedChem Studio (MedChem Studio, 3.0; Simulations Plus, Inc, Lancaster, CA, USA, 2012) according to the user's instructions. Detailed information on drug target prediction for GXJC is provided in Supplementary file S1-section 2 and Supplementary Table S4.

\section{Protein-protein interaction (PPI) information}

The PPI data between putative targets for GXJC and known therapeutic targets for $\mathrm{CHD}$ and depression were obtained from the Human Annotated and Predicted Protein Interaction Database (HAPPI) ${ }^{[24]}$, Reactome ${ }^{[25]}$, the Online Predicted Human Interaction Database (OPHID) $)^{[26]}$, InAct ${ }^{[27]}$, the Human Protein Reference Database (HPRD) ${ }^{[28]}$, the Molecular Interaction Database (MINT) ${ }^{[2]}$, the Database of Interacting Proteins $(D I P)^{[30]}$, and PDZBase ${ }^{[31]}$. Detailed information about these PPI databases is provided in Supplementary Table S5.

\section{Network construction and analysis}

The GXJC putative targets-known therapeutic targets for CHD and depression interaction network was constructed based on the links between GXJC putative targets and known therapeutic targets for CHD and depression and visualized by Navigator software (v2.2.1). Detailed information about this interaction network is provided in Supplementary Table S6.

According to our previous studies ${ }^{[32-34]}$, node degree was calculated to select hubs in the above network, and the hub network was constructed using the interactions among the hubs. Then, four network topological features, including 'Degree,' 'Betweenness,' 'Closeness' and 'K value,' were calculated to evaluate the importance of hubs in the hub network. The definitions of these network topological features are provided in Supplementary file S1-section 3.

\section{Pathway enrichment analysis}

The data obtained from Kyoto Encyclopedia of Genes and Genomes (KEGG; www.genome.jp/kegg/, last updated 2012$10-16)^{[35]}$ were used for pathway enrichment analysis using the DAVID system (http://david.abcc.ncifcrf.gov/home.jsp/, v6.7 $)^{[36]}$.

\section{Results}

Characterization and identification of chemical constituents containing GXJC

The total ion chromatograms (TIC) of the GXJC sample corresponding to the positive and negative signals are shown in Figure 2. Nineteen standard available constituents (Constituents $7,10,11,12,13,17,20,21,23,26,31,40,41,45,47,52,53$ and 62 ), were respectively identified as adenine nucleoside, gallic acid, protocatechuic aldehyde, Dan shensu, crypto tanshinone, benzoic acid, chlorogenic acid, vanillic acid, paeoniflorin, albi florin, lithospermic acid, ginsenoside Rg1, salvianolic acid B, salvianolic acid C, cryptotanshinone, ginsenoside $\mathrm{Rg} 2$, ginsenoside Re and ginsenoside Rg3 by comparing
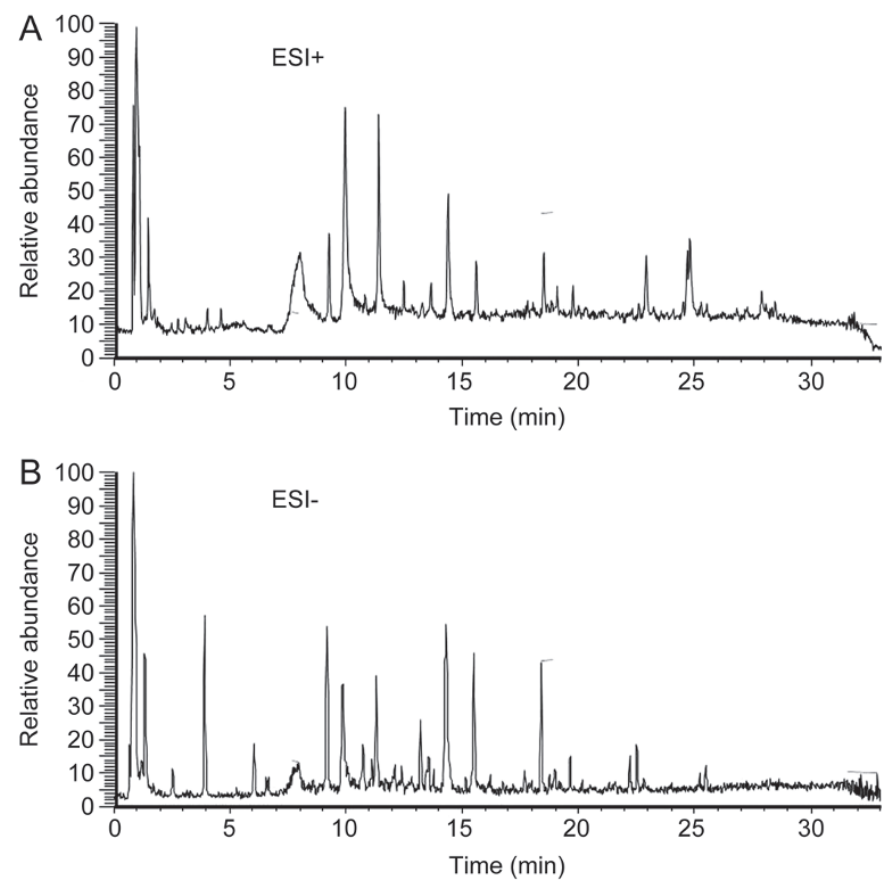

Figure 2. Total ion chromatogram (TIC) of GXJC using UHPLC-LTQ-Orbitrap (A) positive ion mode; (B) negative ion mode.

sample retention times and accurate masses with those of the standards. For the standard unavailable constituents, a series of continuous procedures was used to increase the credibility for structure identification by calculating the molecular formulas using high-resolution and high-accuracy mass spectra, searching in the Chemical Database of GXJC, and determining structure characterization and confirmation using $\mathrm{MS}^{\mathrm{n}}$ mass spectrometry. Overall, a total of 71 constituents, such as ginsenosides, flavonoids and their glycosides, and diterpenoid and monoterpene glycosides, were identified or tentatively characterized with their retention times and $\mathrm{MS}^{\mathrm{n}}$ data. The detailed information of these constituents is summarized in Table 1.

Prediction of the oral absorption and bioavailability of chemical constituents containing GXJC using in silico ADME models

The in silico passive Caco-2 absorption and the pharmacokinetics (PK) explorer modules within the ACD/Percepta software predicted the Papp and F\% for 71 constituents in GXJC. As shown in Table 1, 16 chemical constituents, such as constituent 13 (benzoic acid), constituent 15 (panax ynol), constituent 17 (protocatechuic aldehyde), constituent 21 (vanillic acid), and constituent 23 (paeoniflorin), were identified as having good oral absorption and bioavailability, which suggested these molecules as "drug-likeness" constituents.

\section{Putative targets for GXJC}

A total of 870 putative targets of GXJC were predicted using MedChem Studio (Supplementary Table S4). Among these, there were eight known therapeutic targets for CHD, including ATP-binding cassette sub-family A member 1 (ABCA1), 


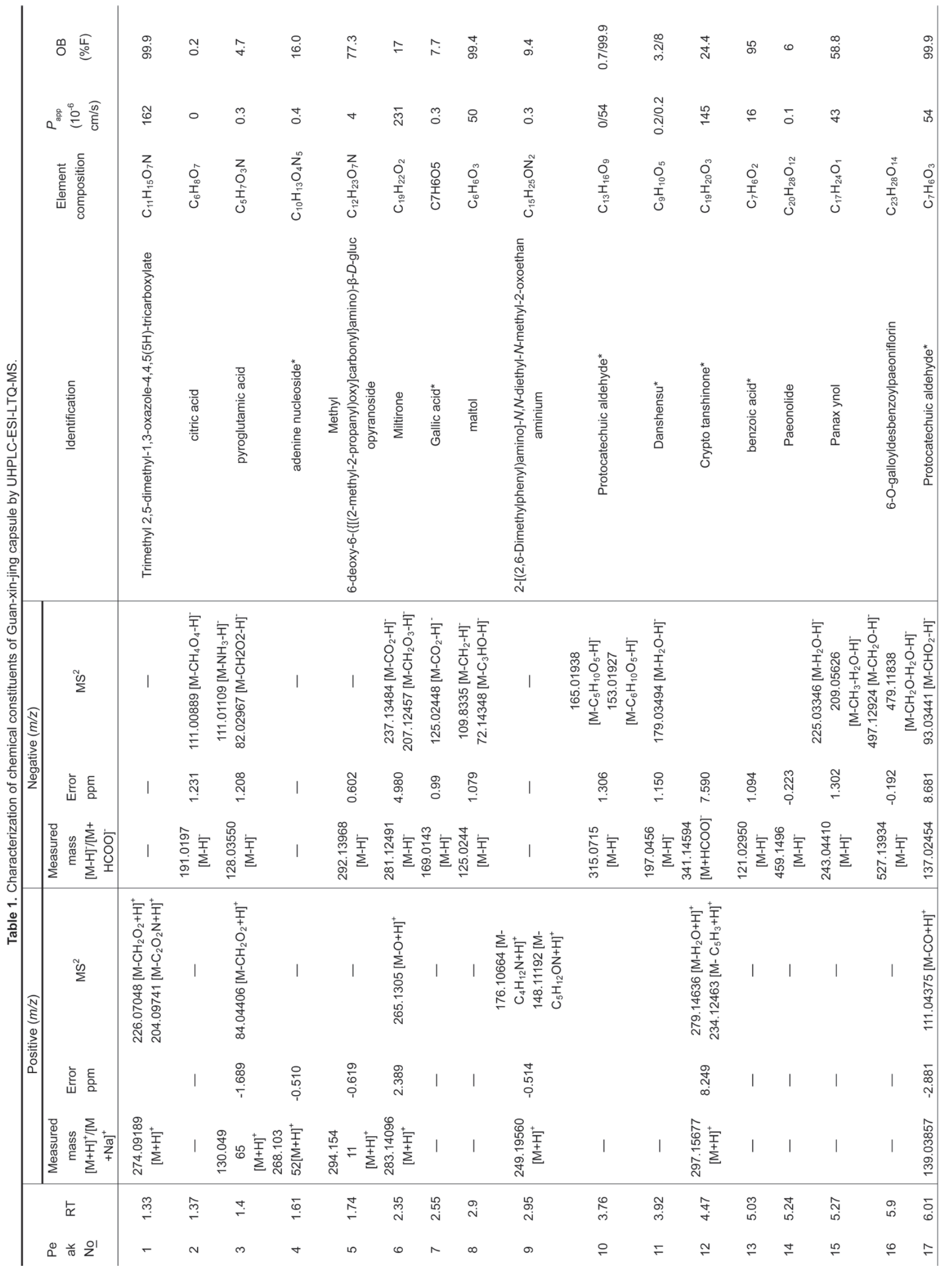




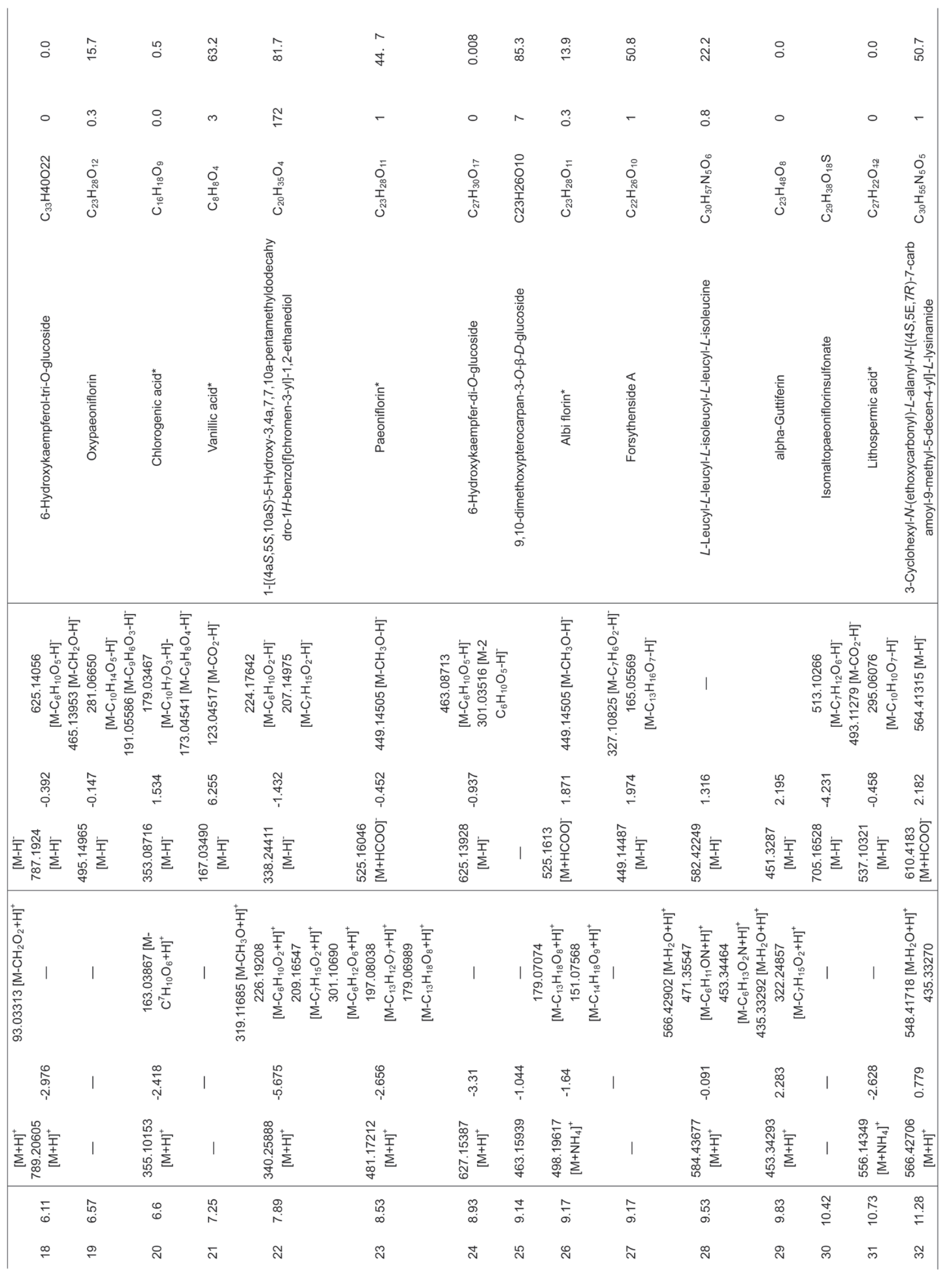




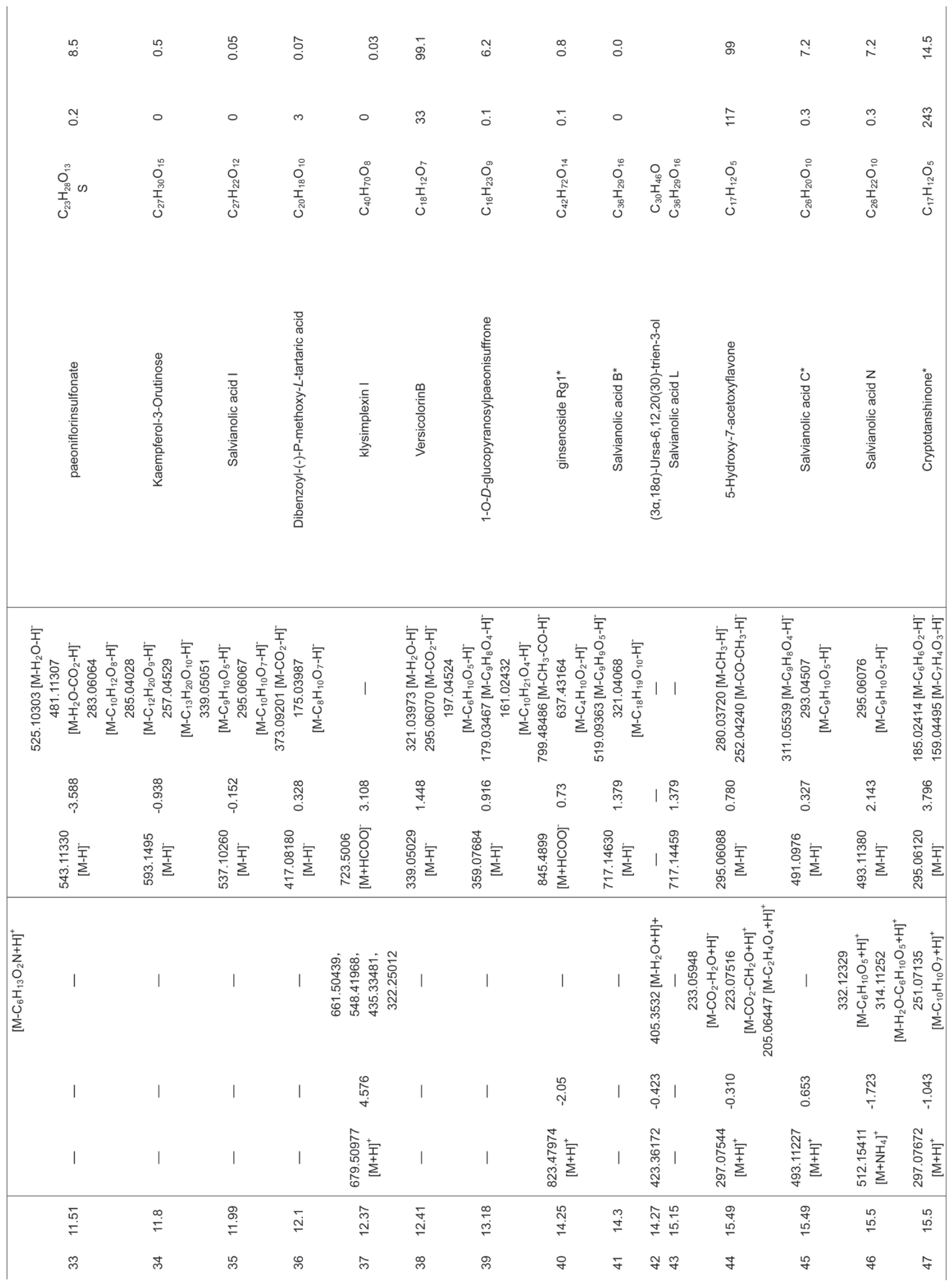




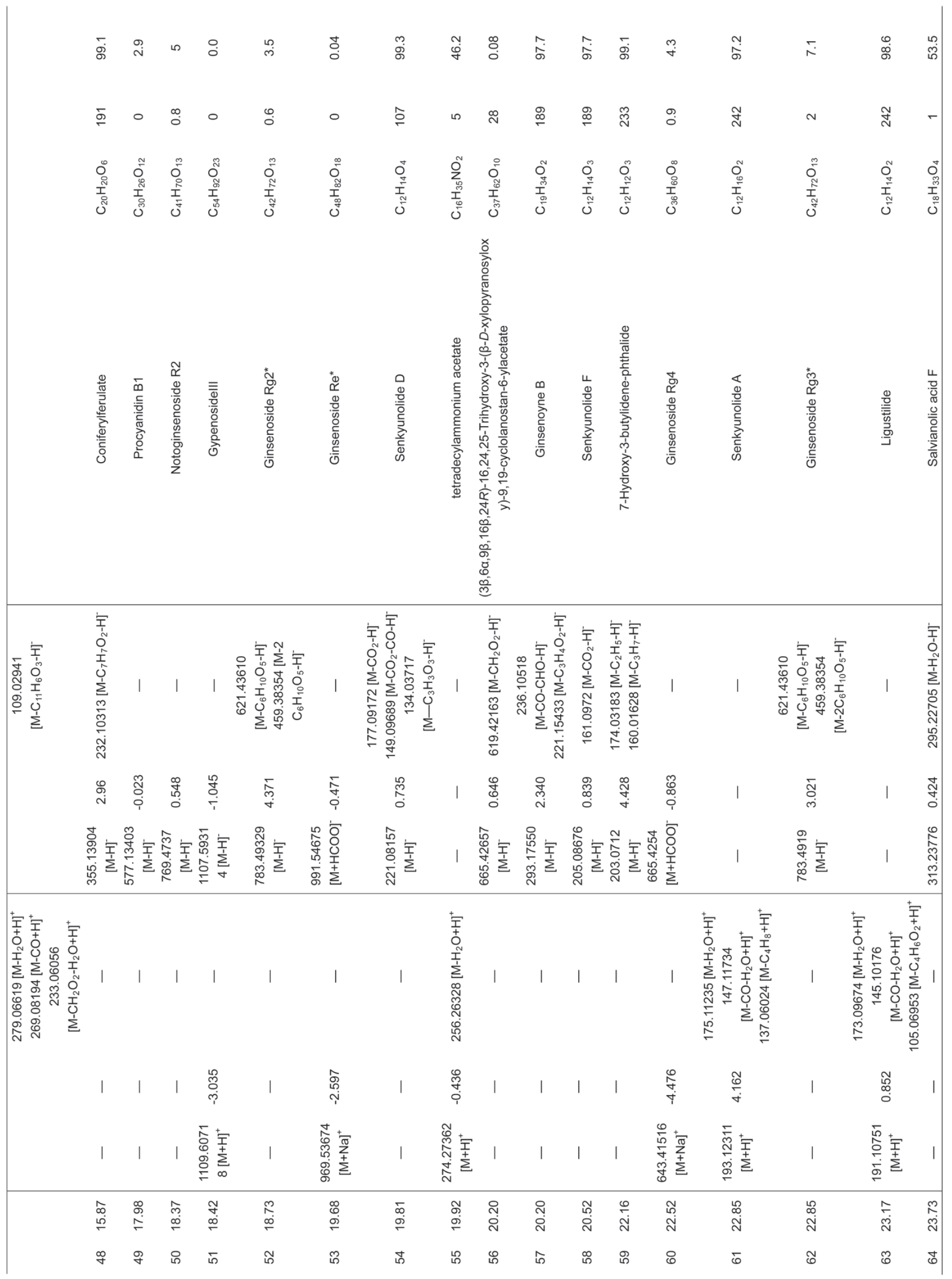




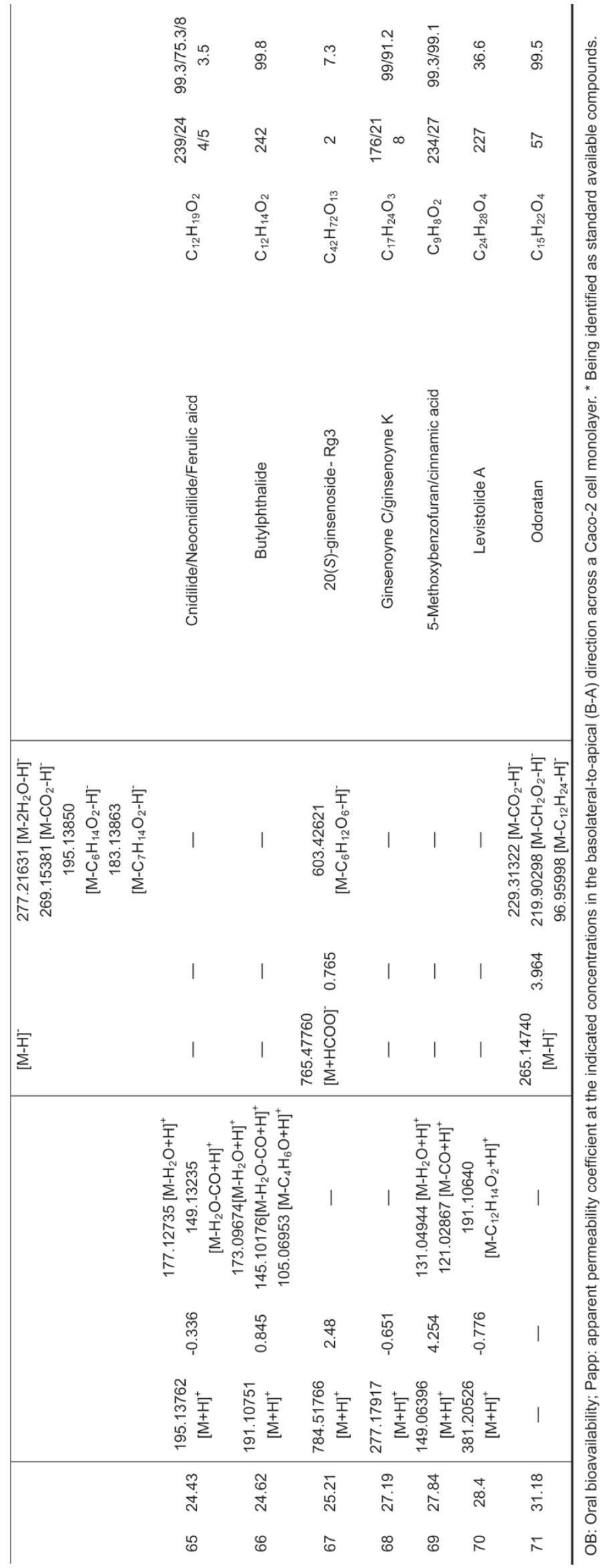

estrogen receptor (ESR1), heparin cofactor 2 (SERPIND1), lysosomal alpha-glucosidase (GAA), ectonucleotide pyrophosphatase/phosphodiesterase family member 1 (ENPP1), G protein-activated inward rectifier potassium channel 4 (KCNJ5), voltage-dependent L-type calcium channel subunit alpha-1D (CACNA1D), 3-beta-hydroxysteroid-delta(8), delta(7)-isomerase (EBP); and eight known therapeutic targets for depression, including 85/88 kDa calcium-independent phospholipase A2 (PLA2G6), phenylalanine-4-hydroxylase (PAH), major prion protein (PRNP), protein kinase C gamma type (PRKCG), platelet-derived growth factor receptor beta (PDGFRB), DNA (cytosine-5)-methyltransferase 1 (DNMT1), cystathionine betasynthase (CBS), and ribonucleoside-diphosphate reductase subunit M2B (RRM2B).

\section{Pharmacological mechanisms of GXJC acting on CHD} complicated with depression

To illustrate the underlying links between drugs and disease, the molecular network-based analysis was performed by building an interaction network of disease-related genes and drug target genes. Here, the GXJC putative target-known $\mathrm{CHD} /$ depression therapeutic target network based on the interactions among putative GXJC targets, known CHD and depression-related targets and other human proteins was constructed. It has been indicated that a node may play a critical role in the network and function as a hub if its degree value was more than 2-fold the median degree of all nodes in a network $^{[37]}$. Therefore, 388 hubs were identified, and the 5107 interactions among them were collected. Detailed information on the hub interaction data in the drug-disease network is provided in Supplementary Table S6.

Next, the major hubs in the hub interaction network were determined by calculating four topological features, including "Degree", "Node betweenness", "Closeness" and "K value". The median values of "Degree", "Node betweenness", "Closeness" and "K value" were 20.00, 0.18, 39.73, and 14.00, respectively. Then, nodes with "Degree" $>20.00$, "Node betweenness" $>0.18$, "Closeness" $>39.73$ and "K value" $>14.00$ were identified as major hubs. As a result, 117 major hubs were selected, and among them, there were 81 putative targets for GXJC (please see detailed information on these four topological features of the 117 major hubs in Supplementary Table S7).

Then, a network of major hubs based on their direct interactions was constructed, and pathway enrichment analysis was performed according to the KEGG pathway database. As shown in Figure 3, these major hubs were divided into three functional modules and involved in the corresponding pathological events during the progression of $\mathrm{CHD}$ and depression. The major hubs in the biggest functional module were mainly involved in five pathways including calcium signaling, VEGF signaling, PDGF signaling, angiotensin mediated activation of JNK and aspirin blocking signaling, as well as long-term depression. In addition, major hubs in the second functional module participated in $\mathrm{T}$ cell receptor signaling pathways and multiple anti-apoptotic pathways and mainly regulated vascular endothelial cell inflammation and vascular endothelial cell 


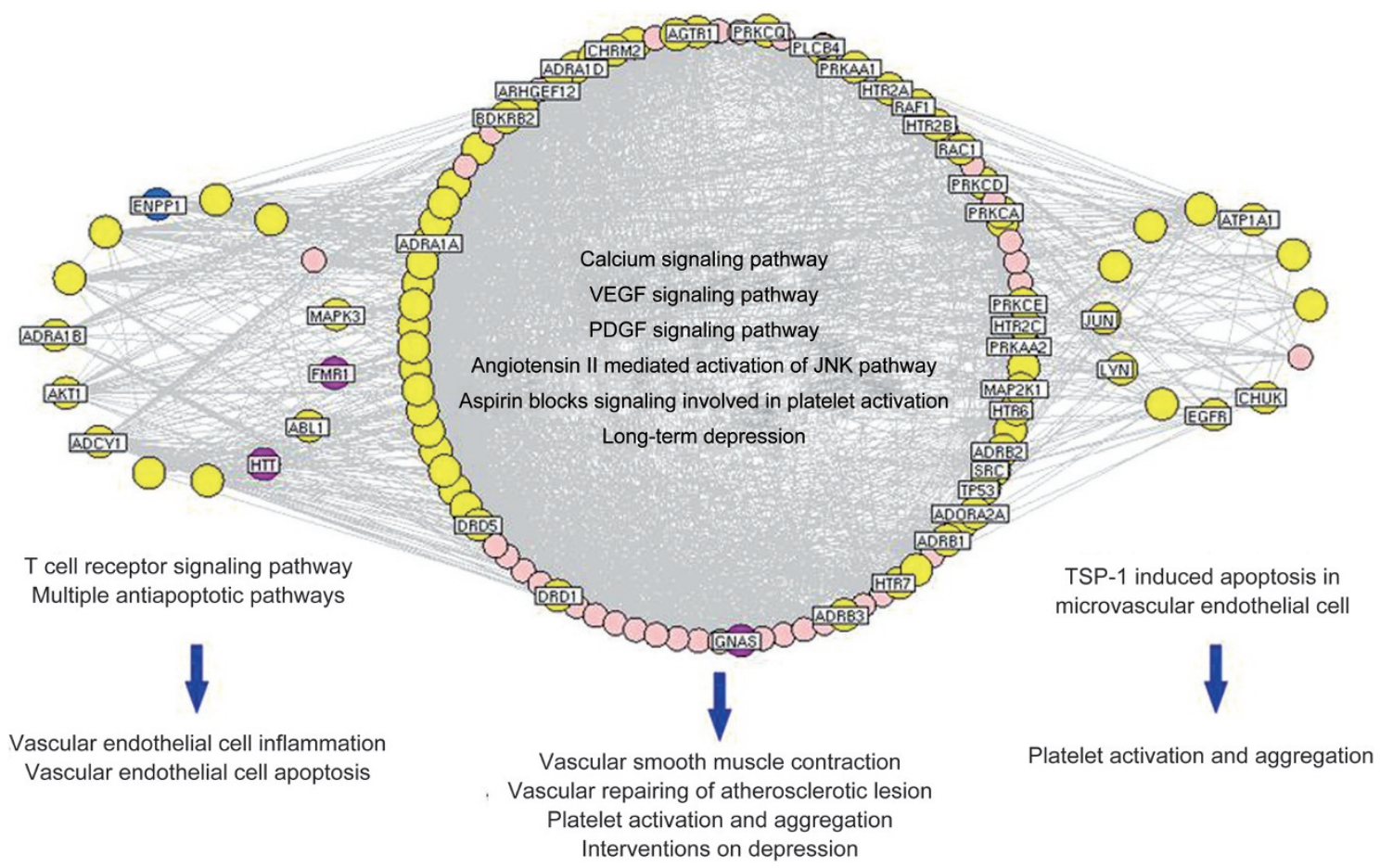

Figure 3. Three functional modules involved in 117 major hubs and the corresponding pathological events during the progression of $\mathrm{CHD}$ complicated with depression. Yellow nodes refer to putative targets for GXJC; blue nodes refer to known therapeutic targets for CHD; purple nodes refer to known therapeutic targets for depression.

apoptosis. Moreover, major hubs in the third functional module were frequently implicated in TSP-1-induced apoptosis in microvascular endothelial cells.

\section{Identification of key active constituents of GXJC acting on CHD} complicated with depression

In the current study, if a drug-like chemical constituent had more than 20 major hub targets, it was regarded as a key active constituent of GXJC acting on CHD complicated with depression. A total of 14 key active constituents were identified: 7 (gallic acid), 17 (protocatechuic aldehyde), 21 (vanillic acid), 35 (salvianolic acid I), 36 (dibenzoyl-(-)-P-methoxy-L-tartaric acid), 48 (coniferyl ferulate), 59 (7-hydroxy-3-butylidenephthalide), 64 (salvianolic acid F), 66 (butylphthalide), 65-3 (ferulic acid), 69-1 (5-methoxybenzofuran), 10 (protocatechuic aldehyde), 11 (dan shensu), and 69-2 (cinnamic acid). Common targets among the key active constituents and the known drugs for the treatment of CHD and depression are listed in Supplementary Table S8. To elucidate the function of key active constituents contained in GXJC, pathway enrichment analysis of the corresponding putative targets was performed. As shown in Figure 4, the major putative targets of the key active constituents were mainly involved into the following 10 pathways: calcium signaling, vascular smooth muscle contraction, VEGF signaling, aspirin blocking signaling, PDGF signaling, T cell receptor signaling, IL-6 signaling, IL-2 signaling, neuroactive ligand-receptor interaction and long-term depression.

\section{Discussion}

In the current study, a total of 16 GXJC drug-like chemical constituents were identified, and 870 genes were predicted to be putative targets of these molecules. According to the topological feature values of the GXJC putative targets, 14 key active constituents were identified because the corresponding putative targets had topological importance in the GXJC putative target-known CHD/depression therapeutic target network and were defined as the candidate targets of GXJC against CHD complicated with depression. Functionally, these candidate targets were significantly involved in several CHD/ depression-related pathways, including repairing pathological vascular changes, reducing platelet aggregation and inflammation, and affecting patient depression.

As a complex chemical system, TCM may be significantly varied because of different origins, sources, harvest time, pretreatments and medicinal material manufacturing ${ }^{[38,39]}$. Therefore, the UHPLC-LTQ-Orbitrap-MSn instrument was utilized to characterize and identify the chemical profiles of GXJC. The oral absorption and bioavailability are two important pharmacokinetic parameters for the constituents of TCM, and growing evidence suggests that chemical constituents with higher Papp values and oral bioavailability (F\%) than $7 \times 10^{6} \mathrm{~cm} / \mathrm{s}$ and $40 \%$, respectively, may have excellent drug-likeness ${ }^{[40-42]}$. The present study employed an in silico passive caco-2 absorption and the pharmacokinetics (PK) explorer modules within the ACD/Percepta software to predict the Papp and F\% for 71 constituents in GXJC and identified 16 chemical constituents 


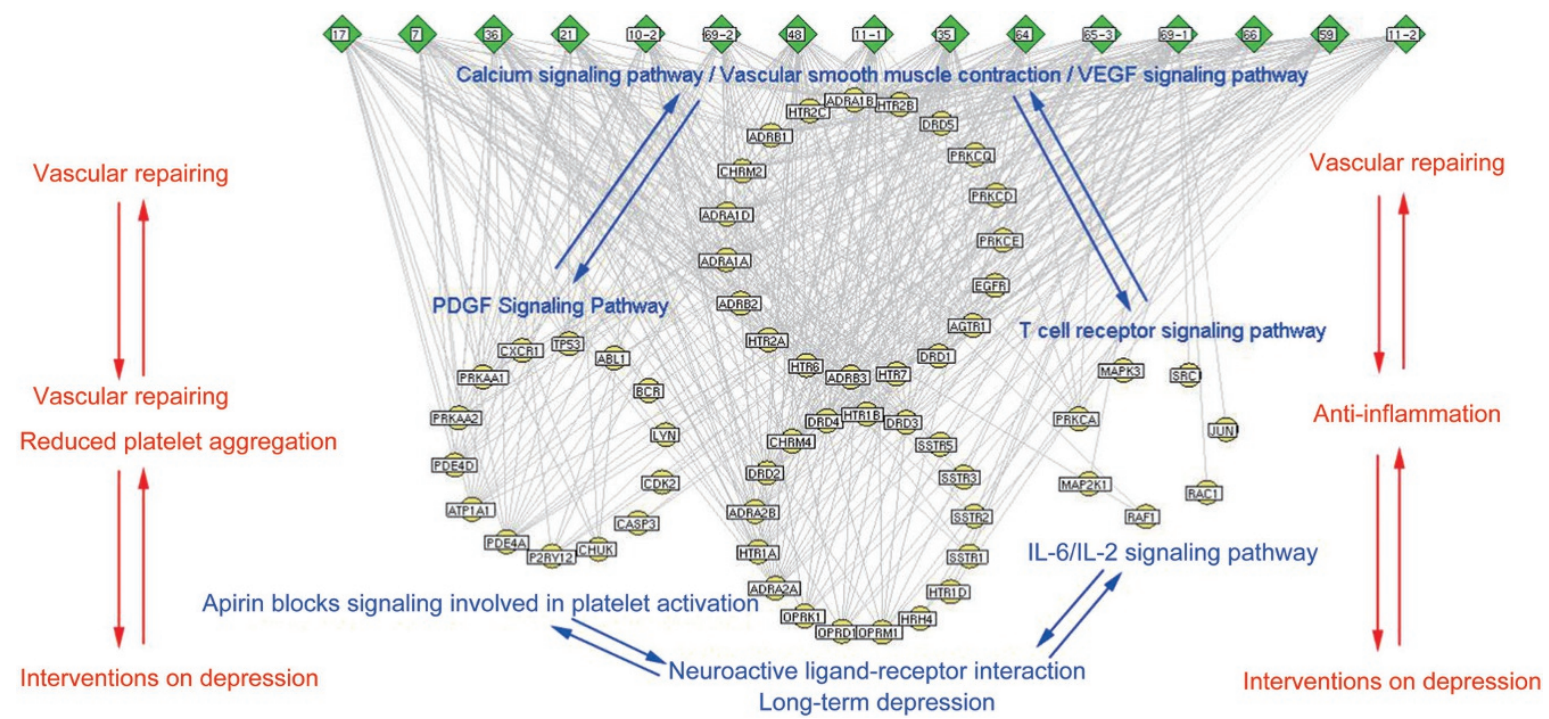

Figure 4. Network of interactions between major chemical constituents contained in GXJC and the corresponding key targets. Green nodes refer to major chemical constituents contained in GXJC; yellow nodes refer to putative targets for GXJC; blue nodes refer to known therapeutic targets for CHD.

with good absorption and drug-likeness.

To investigate the pharmacological mechanisms of GXJC acting on CHD complicated with depression, the putative targets of GXJC were predicted based on the structures and functions of the chemical constituents. After that, the drug targetdisease related gene interaction network was constructed. Among the hub genes of the network, G-protein coupled receptors for 5-hydroxytryptamine, including 5HT1A, 5HT1B, 5HT1D, 5HT2A, 5HT2B, 5HT2C, 5HT4R, 5HT6R, and 5HT7R, regulated the release of 5-hydroxytryptamine, dopamine and acetylcholine in the brain. These genes thereby affect neural activity, perception, cognition and mood, and play a role in arterial vasoconstriction and in the regulation of behavior, including responses to anxiogenic situations and psychoactive substances ${ }^{[43]}$. In addition, ectonucleotide pyrophosphatase/ phosphodiesterase family member 1 (ENPP1), estrogen receptor (ESR1), lysosomal alpha-glucosidase (LYAG), and cAMPdependent protein kinase type I-alpha regulatory subunit (KAP0) are known therapeutic targets for CHD; moreover, KAP0 is also a known depression therapeutic target. ENPP1, an enzyme that produces extracellular pyrophosphate, was reported to be responsible for generalized arterial calcification in infancy or idiopathic infantile arterial calcification ${ }^{[44]}$. The incidence of CHD in premenopausal women is about one-half that in men of similar age and to investigate the underlying reason for this phenomenon, researchers compared the thickness of the coronary intima in women at different ages and determined that ESR1 played a crucial role in coronary intima thickness in young to middle-aged women ${ }^{[45]}$. These findings suggested that GXJC might attenuate CHD complicated with depression through regulating these hub targets.

The therapeutic effects of a Chinese formula are usually decided by its major chemical constituents. Following the identification of the key active constituents of GXJC and the corresponding major putative targets, our functional analysis demonstrated that these major putative targets were significantly associated with calcium signaling, vascular smooth muscle contraction, VEGF signaling, aspirin blocking signaling, PDGF signaling, T cell receptor signaling, IL-6 signaling, IL-2 signaling, neuroactive ligand-receptor interaction and long-term depression, which all play crucial roles in the main pathological processes during CHD progression, including vascular repair, anti-inflammation, and reduction of platelet aggregation ${ }^{[46]}$. Vascular repair is an important treatment strategy for $\mathrm{CHD}$, and protein kinase $\mathrm{C}$ alpha type (PRKCA), epidermal growth factor receptor (EGFR), D (1A) dopamine receptor, type-1 angiotensin II receptor, ras-related C3 botulinum toxin substrate 1 (RAC1), RAF proto-oncogene serine/threonine-protein kinase (RAF1), and proto-oncogene tyrosine-protein kinase Src have been identified as therapeutic targets for vascular injury. Inflammation is a crucial feature of CHD. Indeed, the genes encoding the interleukin 6 receptor (IL6) have been reported to be associated with $\mathrm{CHD}^{[4]}$. Here, the putative targets of GXJC including dual specificity mitogen-activated protein kinase 1 (MAP2K1), transcription factor AP-1 (JUN), mitogen-activated protein kinase 3 (MAPK3), and RAF proto-oncogene serine/threonine-protein kinase (RAF1) were mainly involved in inflammation-related pathways including IL6/IL2/T cell receptor signaling pathway. Platelets play central roles in the development of atherothrombosis and are therefore the target of multiple therapies for $\mathrm{CHD}^{[48]}$. Protein kinase $\mathrm{C}$ alpha type (PRKCA) is a major regulator of various platelet activities and has been reported to regulate platelet activity. The in vivo and in vitro experiment results also indicated that it was required for thrombus formation and could regulate platelet granule secretion ${ }^{[49]}$. More importantly, the key targets of GXJC may also exert intervention effects on depression. These findings suggested that GXJC might be a potential treatment for CHD complicated with depression, which also reflected the purpose of the psycho-cardiology 
treatment.

\section{Conclusions}

This study identified a list of key active constituents of GXJC acting on CHD complicated with depression using an integrative pharmacology-based approach, which combined active chemical constituent identification, drug target prediction and network analysis. This method may offer an efficient way to understand the pharmacological mechanisms of traditional Chinese medicine prescriptions.

Despite the important findings revealed by the current study, there are still several potential limitations. First, the chemical information for some composite constituents of herbs contained in GXJC might be insufficient because of the fragmentary information collected from existing resources. Second, whether the associations between the herbs contained in GXJC and their corresponding targets were direct or indirect was difficult to determine. Third, the interactions between the composite constituents containing GXJC and candidate target genes were obtained via a computational technique. However, it is difficult to verify whether the interactions are positive or negative effects. Last but not least, further experimental studies are needed to validate the findings of this pilot study.

\section{Acknowledgements}

Financial support was provided by the 973 Program of China (№ 2015CB554406), National Key Technology R\&D Program of China (No 2011BAI07B08) and National Natural Science Foundation of China (№ 81473414 and 81673834).

\section{Author contribution}

Yan-qiong ZHANG carried out the experiments and drafted the manuscript; Hai-yu XU conceived of the study, participated in its design and coordination, and helped to draft the manuscript. The other authors, Qiu-yan GUO, Qiu-yue LI, Wei-qiong REN, Shi-huan TANG, Song-song WANG, Ri-xin LIANG, De-feng LI, Yi ZHANG, and Hong-jun YANG, participated in the design of the study and performed the statistical analysis. All authors read and approved the final manuscript.

\section{Supplementary information}

Supplementary information is available at the website of the Acta Pharmacologica Sinica.

\section{References}

1 Nekouei ZK, Yousefy A, Doost HT, Manshaee G, Sadeghei M. Structural model of psychological risk and protective factors affecting on quality of life in patients with coronary heart disease: A psychocardiology model. J Res Med Sci 2014; 19: 90-8.

2 Meng Khoo C, Tai ES. Trends in the incidence and mortality of coronary heart disease in asian pacific region: the Singapore experience. J Atheroscler Thromb 2014; 21: S2-8.

3 Glozier N, Tofler GH, Colquhoun DM, Bunker SJ, Clarke DM, Hare DL, et al. Psychosocial risk factors for coronary heart disease. Med J Aust 2013; 199: 179-80.

4 Januzzi JL Jr, Stern TA, Pasternak RC, DeSanctis RW. The influence of anxiety and depression on outcomes of patients with coronary artery disease. Arch Intern Med 2000; 160: 1913-21.

5 Thombs BD, Bass EB, Ford DE, Stewart KJ, Tsilidis KK, Patel U, et al. Prevalence of depression in survivors of acute myocardial infarction. J Gen Intern Med 2006; 21: 30-8.

6 Carney RM, Freedland KE. Depression, mortality, and medical morbidity in patients with coronary heart disease. Biol Psychiatry 2003; 54: 241-7.

7 Colquhoun DM, Bunker SJ, Clarke DM, Glozier N, Hare DL, Hickie IB, et al. Screening, referral and treatment for depression in patients with coronary heart disease. Med J Aust 2013; 198: 483-4.

8 Francis DK, Bennett NR, Ferguson TS, Hennis AJ, Wilks RJ, Harris EN, et al. Disparities in cardiovascular disease among Caribbean populations: a systematic literature review. BMC Public Health 2015; 15: 828.

9 Fleischer T, Chang TT, Chiang JH, Hsieh CY, Sun MF, Yen HR. Integration of Chinese herbal medicine therapy improves survival of patients with chronic lymphocytic leukemia: a nationwide populationbased cohort study. Medicine (Baltimore) 2016; 95: e3788.

10 Liu J, Liu RL. The potential role of Chinese medicine in ameliorating extra-articular manifestations of rheumatoid arthritis. Chin J Integr Med 2011; 17: 735-7.

11 Wang AL, Chen Z, Luo J, Shang QH, Xu H. Systematic review on randomized controlled trials of coronary heart disease complicated with depression treated with Chinese herbal medicines. Chin J Integr Med 2016; 22: 56-66.

12 Li RP, Yu GH, Li JF, Tang SH. Discussion on relevant theories of "QiBlood-Shen" and formula implication of Guan-Xin-Jing capsule. Chin J Chin Metar Med 2016; 41: 4280-3.

13 National Pharmacopoeia Committee. Chinese pharmacopoeia. Part 1. Beijing: China Medical Science Press 2010: 3-361.

14 Song SJ, Wei FJ, Wei TY, Dong YJ. Clinical observation of Guanxinjing capsule in the treatment of qi deficiency and blood stasis type of coronary heart disease. China J Pharm Economics 2014, S1: 133-4.

15 Jiang JL, Li XJ. Clinical observation of Guanxinjing capsule in the treatment of coronary heart disease complicated with depression. Chin J Tradit Med Sci Technol 2014; 3: 316-7.

16 Yuan SG, Chen R. Mechanism of Guanxinjing capsule acting on psycho-cardiology. J New Chin Med 2013; 12: 176-8.

17 Schardt C, Adams MB, Owens T, Keitz S, Fontelo P. Utilization of the PICO framework to improve searching PubMed for clinical questions. BMC Med Inform Decis Mak 2007; $7: 16$.

18 Lin CH, Chang TT, Sun MF, Chen HY, Tsai FJ, Chang KL, et al. Potent inhibitor design against H1N1 swine influenza: structure-based and molecular dynamics analysis for M2 inhibitors from traditional Chinese medicine database. J Biomol Struct Dyn 2011; 28: 471-82.

19 Chen CY. TCM Database@Taiwan: the world's largest traditional Chinese medicine database for drug screening in silico. PLoS One 2011; 6: e15939.

20 Editorial: ChemSpider-a tool for Natural Products research. Nat Prod Rep 2015; 32: 1163-4.

21 Janicka M, Sztanke M, Sztanke K. Reversed-phase liquid chromatography with octadecylsilyl, immobilized artificial membrane and cholesterol columns in correlation studies with in silico biological descriptors of newly synthesized antiproliferative and analgesic active constituents. J Chromatogr A 2013; 1318: 92-101.

22 Wishart DS, Knox C, Guo AC, Cheng D, Shrivastava S, Tzur D, et al. DrugBank: a knowledgebase for drugs, drug actions and drug targets. Nucleic Acids Res 2008; 36: D901-6.

23 Hamosh A, Scott AF, Amberger JS, Bocchini CA, McKusick VA. Online Mendelian Inheritance in Man (OMIM), a knowledgebase of human genes and genetic disorders. Nucleic Acids Res 2005; 33: D514-7. 
24 Chen JY, Mamidipalli S, Huan T. HAPPI: an online database of comprehensive human annotated and predicted protein interactions. BMC Genomics 2009; 10: S16.

25 Matthews L, Gopinath G, Gillespie M, Caudy M, Croft D, de Bono B, et al. Reactome knowledgebase of human biological pathways and processes. Nucleic Acids Res 2009; 37: D619-22.

26 Brown KR, Jurisica I. Online predicted human interaction database. Bioinformatics 2005; 21: 2076-82.

27 Aranda B, Achuthan P, Alam-Faruque Y, Armean I, Bridge A, Derow C, et al. The IntAct molecular interaction database in 2010. Nucleic Acids Res 2010; 38: D525-31.

28 Keshava Prasad TS, Goel R, Kandasamy K, Keerthikumar S, Kumar S, Mathivanan S, et al. Human Protein Reference Database-2009 update. Nucleic Acids Res 2009; 37: D767-72.

29 Ceol A, Chatr Aryamontri A, Licata L, Peluso D, Briganti L, Perfetto L, et al. MINT, the molecular interaction database: 2009 update. Nucleic Acids Res 2010; 38: D532-9.

30 Lehne B, Schlitt T. Protein-protein interaction databases: keeping up with growing interactomes. Hum Genomics 2009; 3: 291-7.

31 Beuming T, Skrabanek L, Niv MY, Mukherjee P, Weinstein H. PDZBase: a protein-protein interaction database for PDZ-domains. Bioinformatics 2005; 21: 827-8.

32 Guo Q, Zhong M, Xu H, Mao X, Zhang Y, Lin N. A systems biology perspective on the molecular mechanisms underlying the therapeutic effects of Buyang Huanwu Decoction on ischemic stroke. Rejuvenation Res 2015; 18: 313-25.

33 Zhang Y, Guo X, Wang D, Li R, Li X, Xu Y, et al. A systems biologybased investigation into the therapeutic effects of Gansui Banxia Tang on reversing the imbalanced network of hepatocellular carcinoma. Sci Rep 2014; 4: 4154.

34 Zhang Y, Wang D, Tan S, Xu H, Liu C, Lin N. A systems biology-based investigation into the pharmacological mechanisms of Wu Tou Tang acting on rheumatoid arthritis by integrating network analysis. Evid Based Complement Alternat Med 2013; 2013: 548498.

35 Wixon J, Kell D. The Kyoto encyclopedia of genes and genomes-KEGG. Yeast 2000; 17: 48-55.

36 Dennis G Jr, Sherman BT, Hosack DA, Yang J, Gao W, Lane HC, et al. DAVID: Database for annotation, visualization, and integrated discovery. Genome Biol 2003; 4: 3.

37 Li S, Zhang ZQ, Wu LJ, Zhang XG, Li YD, Wang YY. Understanding ZHENG in traditional Chinese medicine in the context of neuroendocrine-immune network. IET Syst Biol 2007; 1: 51-60.

38 Zhang Y, Xu H, Chen X, Chen C, Wang H, Meng F, et al. Simultaneous quantification of 17 constituents from Yuanhu Zhitong tablet using rapid resolution liquid chromatography coupled with a triple quadrupole electrospray tandem mass spectrometry. J Pharm Biomed Anal 2011; 56: 497-504.

39 Wang S, Xu H, Ma Y, Wang X, Shi Y, Huang B, et al. Characterization and rapid identification of chemical constituents of NaoXinTong capsules by UHPLC-linear ion trap/Orbitrap mass spectrometry. J Pharm Biomed Anal 2015; 111: 104-18.

40 Tao Y, Xu H, Wang S, Wang B, Zhang Y, Wang W, et al. Identification of the absorbed constituents after oral administration of Yuanhu Zhitong prescription extract and its pharmacokinetic study by rapid resolution liquid chromatography/quadrupole time-of-flight. J Chromatogr B Analyt Technol Biomed Life Sci 2013; 935: 1-9.

41 Yazdanian M, Glynn SL, Wright JL, Hawi A. Correlating partitioning and caco-2 cell permeability of structurally diverse small molecular weight constituents. Pharm Res 1998; 15: 1490-4.

$42 \mathrm{Xu} \mathrm{H}$, Shi Y, Zhang Y, Jia Q, Li D, Zhang Y, et al. Identification of key active constituents of Buchang Naoxintong capsules with therapeutic effects against ischemic stroke by using an integrative pharmacologybased approach. Mol Biosyst 2016; 12: 233-45.

43 Harrington MA, Shaw K, Zhong P, Ciaranello RD. Agonist-induced desensitization and loss of high-affinity binding sites of stably expressed human 5- $\mathrm{HT}_{1 \mathrm{~A}}$ receptors. J Pharmacol Exp 1994; 268 : 1098-106.

44 Hofmann Bowman MA, McNally EM. Genetic pathways of vascular calcification. Trends Cardiovasc Med 2012; 22: 93-8.

45 Henttonen AT, Kortelainen ML, Kunnas TA, Nikkari ST. Estrogen receptor-1 genotype is related to coronary intima thickness in young to middle-aged women. Scand J Clin Lab Invest 2007; 67: 380-6.

46 Vital SA, Becker F, Holloway PM, Russell J, Perretti M, Granger DN, et al. Formyl-peptide receptor 2/3/Lipoxin A4 receptor regulates neutrophil-platelet aggregation and attenuates cerebral inflammation: impact for therapy in cardiovascular disease. Circulation 2016; 133 : 2169-79.

47 Bullock-Palmer RP. Prevention, detection and management of coronary artery disease in minority females. Ethn Dis 2015; 25 : 499-506.

48 Kessler T, Vilne B, Schunkert $H$. The impact of genome-wide association studies on the pathophysiology and therapy of cardiovascular disease. EMBO Mol Med 2016; 8: 688-701.

49 Meadows TA, Bhatt DL. Clinical aspects of platelet inhibitors and thrombus formation. Circ Res 2007; 100: 1261-75. 